\title{
Considerations for the Design of Antibody-Based Therapeutics
}

\author{
Harapriya Sahoo* \\ Department of Microbiology, Utkal University, Bhubaneswar, Odisha, India
}

\section{Commentary}

Antibody-based based proteins have become a significant class of biologic therapeutics, to a great extent because of the soundness, particularity, and flexibility of the immunizer structure. In fact, antibodies not just have the inborn capacity to tie the two antigens and endogenous resistant receptors yet additionally have demonstrated amazingly managable to protein designing. Consequently, a few subordinates of the monoclonal neutralizer design, including bispecific antibodies, counter acting agent drug forms, and immunizer pieces, have exhibited viability for treating human infection, especially in the fields of immunology and oncology. Surveyed here are contemplations for the plan of counter acting agent based therapeutics, including immunological setting, remedial systems, and designing procedures. In the first place, qualities of antibodies are presented, with accentuation on primary areas, practically significant receptors, isotypic and allotypic contrasts, and alterations like glycosylation. Then, at that point, parts of helpful antibody configuration are talked about, including ID of antigen-explicit variable areas, decision of articulation framework, utilization of multi specific organizations, and plan of immunizer subordinates dependent on discontinuity, oligomerization, or formation to other utilitarian moieties. At long last, procedures to upgrade counter acting agent work through protein designing are surveyed while featuring the effect of principal biophysical properties on protein develop ability.

The main remedial monoclonal neutralizer, muromonab-CD3 (OKT3), was endorsed by the Food and Drug Administration in 1985 to forestall dismissal of kidney, heart, and liver transfers.

In an ordinary instrument for counter acting agent based therapeutics, OKT3 ties to and represses CD3 on the T-cell receptor complex to forestall have $T$ cells from being initiated against unfamiliar antigens on the relocated tissue. In spite of the fact that OKT3 demonstrated successful for forestalling host-versus-unite sickness, the neutralizer itself gets an insusceptible reaction, bringing about its sped up leeway. The beginning of this invulnerable response has been followed to nonhuman groupings on OKT3, a murine neutralizer. Resulting ages of restorative antibodies have acculturated the amino corrosive succession of mouse antibodies to fanciful, refined, and completely human. This acculturation of grouping to forestall immunogenicity is only one illustration of how immunizer based therapeutics have been worked on as the decades progressed. Truth be told, each piece of the immunizer structure has been deliberately adjusted to change organic impacts and work on clinical results.

Neutralizer therapeutics address the quickest developing class of medications available, to a great extent because of normally ideal credits like particularity, intensity, and metabolic dependability. Information on humoral immunology and advances in protein designing have additionally added to the improvement of these significant medications. Right now, 76 neutralizer based therapeutics are utilized in the facility, with close to as numerous in late phases of clinical preliminaries.

The most productive utilizations of antibodies lie in the fields of oncology (where underlying effector capacities help to dispose of tumor cells) and immunology (where hindrance of fiery pathways is valuable in treating

*Address for Correspondence: Sahoo H, Department of Microbiology, Utkal University, Bhubaneswar, Odisha, India, E-mail: harapriyas97@gmail.com

Copyright: (C) 2021 Sahoo H. This is an open-access article distributed under the terms of the Creative Commons Attribution License, which permits unrestricted use, distribution, and reproduction in any medium, provided the original author and source are credited.

Received 22 July 2021; Accepted 30 July 2021; Published 07 August 2021 autoimmunity). Over the long haul, progressively creative neutralizer subordinates have supplanted the standard monoclonal immune response to address the complex pathobiology of illness and refine existing treatments.

When planning immunizer based therapeutics, various components should be thought of, with each factor straightforwardly affecting protein structure and resulting impacts on natural and helpful capacities. For instance, the decision of designated antigen and neutralizer age methodology influences the essential and tertiary construction of the immune response variable areas. Contrasts in this space of the protein sway the idea of the counter acting agent antigen cooperation, including explicitness, partiality, and regardless of whether the limiting occasion is enacting or inhibitory. These natural properties, thus, decide clinical properties like power and helpful record. Along these lines, factors like counter acting agent subclass and allotype influence the construction of the steady locales, which thusly impacts restricting to crystallizable part $(\mathrm{Fc})$ receptors ( $\mathrm{FcRs}$ ) significant for effector capacity and serum half-life. Hence, a few determinants should be viewed as while making new neutralizer based therapeutics. Albeit particular primary highlights have covering useful outcomes, antibodies can be planned in a measured style to join all ideal highlights into a solitary improved atom. In this survey, different plan components of helpful antibodies are talked about, alongside their effects on structure and organic and clinical capacities. The point is to cover the wide degree of plan methodologies and designing alternatives accessible as opposed to comprehensively examine the writing on some random subject. Subsequently, more engaged audits have been refered to for intensive conversation of individual plan components.

The original of antibody therapeutics zeroed in essentially on explicit restricting of sub-atomic focuses to evoke straightforward inhibitory components. While these early atoms set up huge particles as a legitimate class of medications, they didn't completely gain by all parts of the counter acting agent stage. All the more as of late, helpful systems have been tweaked dependent on the sort of antigen as well as by antigen partiality, valency, and epitope. Utilization of various antibody subclasses considers adjusting of PK and effector work as a result of differential restricting to endogenous FcRs and supplement proteins. In the meantime, little antigen-restricting areas can be utilized to take out effector work, multimerizing antibodies increment supplement obsession, and counter acting agent combinations impart different proteins with great properties of the immunizer system. Formation of cytotoxic specialists to antibodies takes into account explicit conveyance of payloads to tumors, while multi specific antibodies give novel systems that expansion particularity and work with conveyance to truly recalcitrant compartments. In corresponding with these system advancements, counter acting agent designing considers joining of amino corrosive and glycan changes that specifically modify natural and actual properties.

Taking everything into account, the humoral safe reaction makes astoundingly complex counter acting agent particles with the capacity to tie the two antigens and components of the versatile invulnerable framework. This neutralizer design has demonstrated to be amazingly amiable to protein designing, which takes into account secluded plan of underlying areas that best coordinate the ideal helpful capacities. With expanded comprehension of immunobiology and the proceeded with improvement of sub-atomic natural strategies, the opportunities for immunizer based therapeutics are limited exclusively by the extent of human creativity.

How to cite this article: Sahoo, Harapriya. "Considerations for the Design of Antibody-Based Therapeutics." Pharmaceut Reg Affairs 10 (2021): 258. 